\title{
ㄷำ1
}

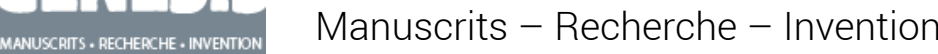

43 | 2016

Bande dessinée

\section{La fabrique de la bande dessinée}

Documents d'étape d'un processus étagé et mouvant

\section{Thierry Groensteen}

\section{CpenEdition}

Journals

Édition électronique

URL : http://journals.openedition.org/genesis/1702

DOI : 10.4000/genesis. 1702

ISSN : 2268-1590

Éditeur :

Presses universitaires de Paris Sorbonne (PUPS), Société internationale de génétique artistique littéraire et scientifique (SIGALES)

\section{Édition imprimée}

Date de publication : 12 décembre 2016

Pagination : 43-50

ISBN : 9791023105490

ISSN : $1167-5101$

\section{Référence électronique}

Thierry Groensteen, "La fabrique de la bande dessinée », Genesis [En ligne], 43 | 2016, mis en ligne le 23 novembre 2017, consulté le 20 avril 2019. URL : http://journals.openedition.org/genesis/1702 ; DOI : 10.4000/genesis.1702 


\section{La fabrique de la bande dessinée Documents d'étape d'un processus étagé et mouvant}

Thierry Groensteen

I

1 fut un temps où ce que l'on appelle communément bande dessinée était souvent taxé de sous- ou d'infra-littérature, au mieux relayé dans le second rayon, celui des «mauvais genres ». N'en déplaise à quelques derniers réfractaires, il est reconnu, désormais, que la bande dessinée est une littérature en soi, une littérature à part entière, susceptible d'investir tous les genres, de donner carrière à toutes les ambitions : fictionnelle, documentaire, introspective, poétique, etc.

Son «inventeur» au XIXe siècle, Rodolphe Töpffer (1799-1846), la qualifiait déjà de littérature en estampes. Au début des années soixante, animé par un souci de légitimation, lepremier «club des bandes dessinées» choisit de se rebaptiser Centre d'étude des littératures d'expression graphique (CELEG). Plus près de nous, le chercheur Harry Morgan a donné à son grand essai théorique le titre de Principes des littératures dessinées ${ }^{1}$. Et la vogue récente $\mathrm{du}$ « roman graphique» - si difficile à définir que soit cette catégorie ${ }^{2}$ - est venue enfoncer le clou.

Quand nous disons que la bande dessinée est une littérature, nous signifions que son support est l'imprimé (ou, désormais, quelquefois, l'écran), que son mode d'appréhension est la lecture, que le champ se structure en collections, genres, séries, et qu'il participe de la vie de l'édition.

Mais la bande dessinée est aussi un art visuel, un art du dessin. C'est bien ainsi que la considèrent désormais le marché de l'art et l'ensemble de ses acteurs (commissaires-priseurs, directeurs de musées, galeristes, collectionneurs...). Et parmi les chercheurs qui ont étudié les spécificités de son langage, la majorité sans doute considère aujourd'hui, comme j'ai pu l'écrire, que l'image prévaut sur le texte parce que «l'essentiel de la production du sens s'effectue à travers elle ${ }^{3} »$. Du reste, il existe une riche tradition de bandes dessinées appelées «muettes», qui alignent des images sans aucun recours au verbal.

La bande dessinée est donc frappée d'une ambiguïté constitutive, ou plutôt d'une hybridité ontologique. Non pas parce qu'elle associe nécessairement le verbal et l'iconique, mais parce qu'elle se situe au confluent de la littérature et des arts visuels, participant indissociablement des deux.

Cette hybridité n'est pas sans conséquence sur le processus d'élaboration des œuvres. Concrètement, une bande dessinée peut être l'œuvre d'un auteur unique, réalisant et concevant tout à lui seul, sans aide extérieure, ou bien être le résultat d'une collaboration entre un spécialiste

1. Harry Morgan, Principes des littératures dessinées, Angoulême, Éditions de l'An 2, 2003.

2. Voir à ce sujet mon article «Roman graphique», «Dictionnaire esthétique et thématique de la bande dessinée», revue en ligne Neuvième Art 2.0, publié en septembre $2012:<$ http://neuviemeart.citebd.org/spip.php?article448 >. 3. Voir Thierry Groensteen, Système de la bande dessinée, Paris, PUF, 1999, p. 10. 
du récit (le scénariste) et un spécialiste de l'image (le dessinateur). D'autres collaborateurs spécialisés peuvent aussi intervenir : assistants, lettreur, coloriste, graphiste, informaticien... Au Japon, le rédacteur en chef du magazine dans lequel une bande dessinée est prépubliée collabore souvent étroitement avec l'auteur principal, jouant un rôle de conseiller très actif. Certaines bandes dessinées (très minoritaires) sont le produit d'un véritable travail de studio cette organisation tayloriste répondant à la nécessité de respecter un rythme de production élevé.

Ce qui est premier dans le processus d'invention, c'est, en principe, le scénario. De même qu'un cinéaste ne se met généralement pas au travail sans savoir ce qu'il veut raconter et ce qu'il va devoir tourner, un dessinateur a besoin de connaître le récit dont il va s'emparer. Dans certains cas, le sujet va d'ailleurs exiger de lui des recherches documentaires préalables, des lectures, des repérages.

Il arrive, sans doute, qu' une bande dessinée obéisse aux lois de l'improvisation, c'est-à-dire à sa non-loi : au lieu de suivre ce document prescriptif qu'est le scénario, un dessinateur peut entreprendre une page, ou un récit plus long (par exemple les quatre-vingt-dix-huit pages du Garage hermétique de Jerry Cornelius, s'il s'appelle Mœbius, ou les cinq cents pages de Lapinot et les carottes de Patagonie, s'il se nomme Lewis Trondheim) en se laissant porter par le dessin même, en obéissant à ses suggestions, en suivant les caprices de son imagination et en faisant porter à conséquence les «accidents du crayon» (pour reprendre une expression d'Hergé) $)^{4}$. Le cas demeure rare.

Les scénaristes peuvent être définis comme des hommes de mots. Le document qu'ils remettent au dessinateur est un écrit, qui comporte deux sortes d'énoncés : des descriptions, plus ou moins détaillées, de ce qui devra être représenté, d'une part, et les répliques constituant le dialogue, d'autre part. Pour chaque case, donc : ce qui sera montré $v s$ ce qui sera dit verbalement (étant entendu que le montré est lui-même un dit). Les descriptions sont destinées au dessinateur et à lui seul; il lui reviendra de les convertir en images - de faire de la chenille un papillon, selon une métaphore un peu usée. Le dialogue, lui, fera partie de l'œuvre finale, il en constituera la «bande texte» (comme on parle de «bande son» au cinéma).

Pourtant certains scénaristes ont eux-mêmes été dessinateurs, ou le demeurent à l'occasion, ou encore sont avant tout des dessinateurs qui, ponctuellement, proposent des sujets à des confrères. Dans ce cas, il est fréquent que le scénario communiqué à celui qui a en charge la mise en dessin de l'histoire soit lui-même déjà dessiné : une suite de croquis rapides, indicatifs, un «storyboard 5 » qui, mieux que de longs discours, fera comprendre les intentions. Cette version opérationnelle du scénario peut avoir été précédée d'une phase d'écriture proprement dite : prise de notes, rédaction d'un synopsis destiné à convaincre l'éditeur ou à fixer ses propres idées, séquencier, etc. Mais le dessinateur va travailler à partir d'un état déjà dessiné du projet. L'opération de traduction d'un système sémiotique dans un autre disparaît alors : il s'agit de passer d'une suite de dessins approximatifs, inaboutis, à une autre suite de dessins plus construits, plus précis, avec une mise en scène et des cadrages plus étudiés, des décors plus fouillés (s'il y a lieu), des expressions plus travaillées, etc.

4. Pour plus de détails, lire mon article «Improvisation», «Dictionnaire esthétique et thématique de la bande dessinée», op. cit., mars $2014:$ : <http://neuviemeart.citebd.org/spip.php?article751>.

5. Sur la notion de storyboard appliquée à la bande dessinée, je renvoie à mon article «Du cinéma dessiné à la bande dessinée», Storyboard, le cinéma dessiné, Crisnée (Belgique), Yellow Now, avril 1992, p. 171-183. 
La phase de scénarisation est avant tout d'invention, tandis que la phase de mise en dessin, elle, est surtout d'exécution - même si chaque élément du récit peut à tout moment être discuté et remis en cause.

Si le scénariste ne dessine pas lui-même, cela ne signifie pas pour autant qu'il n'est pas préoccupé d'images. En 1988, Benoît Peeters, scénariste des Cités obscures pour François Schuiten, écrivait : «De plus en plus, il me semble que le bon scénario de bande dessinée est moins celui qui approche d'un en-soi de la perfection narrative, du reste bien difficile à définir, que celui qui propose une puissante machine à dessiner. [...] la première tâche du scénariste serait donc ce que l'on pourrait nommer l'écriture de l'autre, c'est-à-dire la mise en place des conditions qui vont favoriser l'émergence de ces objets mystérieux et fragiles : des images6.» Un vif intérêt et une longue familiarité avec la bande dessinée amènent généralement les scénaristes à penser d'emblée en images et à écrire en vue de l'image.

Dans quelques rares cas demeurés célèbres, il est arrivé que la préséance chronologique entre les phases d'écriture et de dessin soit bouleversée.

Scénariste de L'Incal pour Mœbius, Alexandro Jodorowsky préférait lui raconter oralement l'histoire, en la mimant. Sur la base des notes et des croquis pris au cours de ces séances de travail, le dessinateur se mettait au travail, le Chilien réintervenant dans un deuxième temps pour préciser les dialogues.

Stan Lee et Jack Kirby sont deux noms légendaires pour les amateurs de comic books de super-héros. On leur doit notamment la création des Quatre Fantastiques, du Surfer d'Argent, de Hulk et de Thor. Leur collaboration obéissait à un protocole singulier : après que les deux hommes s'étaient mis d'accord sur les grandes lignes du récit à venir, c'est le dessinateur, Kirby, qui, sans plus d'indications, réalisait les pages, au crayon, sous une forme purement graphique, endossant ainsi la responsabilité du détail des péripéties et du découpage. Lee reprenait ensuite la main pour rédiger le texte, en se basant sur les quelques annotations déjà inscrites dans les marges.

À la lumière de tout ce qui précède, on comprend que la notion de «manuscrit autographe» s'applique mal à la bande dessinée. Quand ce ne serait que pour les cinq raisons qui suivent.

1. L'éditeur d'un texte (roman, essai) est responsable de sa mise en forme; il définit un format, choisit une police de caractères, un corps, un interlignage. La répartition du texte dans les pages est le résultat de ces choix éditoriaux et échappe aux prérogatives de l'auteur. Dans le cas de la bande dessinée, les planches remises à l'éditeur (aujourd'hui presque toujours sous la forme de fichiers numériques) sont des matrices qui seront reproduites telles quelles. Leur ordonnance, leur composition, leur équilibre font partie des paramètres visuels pensés et réglés par le dessinateur.

2. Un manuscrit autographe peut acquérir de la valeur sur le marché spécialisé. Cette valeur lui est conférée par la notoriété de l'auteur. Une planche de bande dessinée, elle, est une œuvre plastique qui existe physiquement en dehors de l'œuvre dont elle participe, qui peut être appréciée pour elle-même, pour ses qualités artistiques; autant que la réputation du dessinateur, ce sont ces qualités propres qui lui confèrent une valeur marchande et qui en font un objet de collection convoité.

6. Benoît Peeters, «L'écriture de l'autre», Les Cahiers de la bande dessinée, n 81, juin 1988, p. 40-43. 
3. Il faut, pour un manuscrit autographe, faire expertiser l'écriture pour s'assurer de son authenticité. S'agissant d'une planche, le style du dessinateur est immédiatement reconnu par l'amateur et, jusqu'à présent, la contrefaçon n'existe pas.

4. Quand bien même elle porte la signature du dessinateur, la planche originale peut n'être pas pleinement autographe, au sens où elle est souvent le résultat d'un travail en collaboration. Le scénariste peut légitimement se prévaloir d'une partie de sa paternité. (Un débat agite d'ailleurs la profession, sur le point de savoir si le scénariste doit être intéressé au bénéfice d'une vente éventuelle de l'original, et dans quelle proportion; certains ont avancé l'idée que le dessinateur pourrait faire don au scénariste d'un certain nombre de planches dont celui-ci disposerait librement. La question n'est pas tranchée.) Des assistants ont pu y prêter la main.

5. La planche originale témoigne du stade terminal d'un processus de création qui a pu auparavant s'incarner dans une multiplicité de documents : notes, carnets ${ }^{7}$, scénario, découpage, brouillon, storyboard, esquisses, crayonné... Pour autant, la planche originale est encore, le plus fréquemment, un document incomplet, comme le rappelle Jean-Pierre Mercier, conseiller scientifique à la Cité internationale de la bande dessinée et de l'image : «elle est souvent en noir et blanc, alors que la page dans sa version finale est imprimée en couleurs; parfois, les bulles de texte sont vides, car le texte est calligraphié sur support séparé8».

C'est pourquoi la génétique de la bande dessinée ne peut se borner à l'examen d'un introuvable manuscrit, mais doit être pensée en termes de procès : elle ne peut consister qu'en une étude des phases successives de la création et de l'ensemble des documents d'étapes, des «avant-textes »-dans la mesure où ceux-ci ont été conservés. En outre, la première version publiée n'est pas nécessairement définitive. Au moment du passage de la prépublication dans un support presse à la publication sous la forme d'un ouvrage de librairie, ou à l'occasion d'une réédition ultérieure, le ou les auteur(s) peut (peuvent) procéder à des changements. Le champ qui s'ouvre à la recherche s'étend donc en amont et en aval de la première version imprimée : depuis les recherches, tâtonnements, versions abandonnées des premiers temps jusqu'aux variantes, refontes, repentirs et ajouts tardifs. L'étude portera soit sur les mécanismes de l'invention, soit sur le jeu des écarts entre les versions successives.

Entre la réception du scénario et la réalisation de la planche, le dessinateur exécute habituellement des esquisses. Contrairement à ce que l'on pourrait croire, ce ne sont pas toujours les images les plus réalistes, les plus détaillées, qui s'appuient sur une quantité importante d'esquisses. Ainsi, quiconque lit une bande dessinée de Claire Bretécher a l'impression qu'elle a dessiné vite, au fil de la plume. Il n'en est rien : chacune de ses images a été précédée par des croquis préparatoires, quelquefois nombreux, à la recherche des attitudes les plus adéquates, les plus expressives. De même, le style volontairement simplifié - presque diagrammatique de Maus, le chef-d'œuvre d'Art Spiegelman, ne doit pas nous abuser : la mise au point de

7. Pour les avoir fait acquérir par le Musée de la Bande dessinée, j’ai pu m’appuyer sur les carnets de travail de Martin Vaughn-James pour analyser son fameux «roman visuel» expérimental, La Cage. Voir Thierry Groensteen, La Construction de La Cage. Autopsie d'un roman visuel, Bruxelles, Les Impressions nouvelles, 2002. Texte repris dans la nouvelle édition de La Cage proposée en 2010 par le même éditeur.

8. Jean-Pierre Mercier, «Un objet incertain : l’original de bande dessinée », Support/Tracé, n 12, 1er trim. 2013, p. 25-31 ; cit. p. 25-26. 
chaque page a nécessité de nombreuses recherches graphiques, dont l'auteur a du reste donné un large échantillon dans MetaMaus 9 .

Il existe deux sortes d'esquisses : celles qui anticipent une image précise, et celles qui mettent à l'épreuve l'ordonnance globale de la page. Les premières font émerger un dessin; les secondes définissent, en juxtaposant des formes géométriques, la configuration du «multicadre», assignant à chaque vignette une position, une forme et une superficie données. Ce que les dessinateurs désignent fréquemment comme le storyboard croise ces deux axes : il s'agit d'un brouillon complet du récit projeté, qui répartit la matière narrative dans les pages disponibles, fixant un premier état du découpage, de la mise en page, du rythme, mais qui ébauche déjà, en même temps, la composition de chacune des images. D'autres esquisses viendront ensuite préciser, reprendre, peaufiner, sinon toutes les vignettes, du moins celles qui nécessitent une recherche graphique plus poussée.

L'étape préparatoire la plus aboutie est habituellement (ou du moins était, avant que ne se répande l'outil informatique) celle du crayonné. Le dessinateur réalise, au format d'exécution qu'il juge le plus confortable (pour certains c'est une feuille A4, pour d'autres une feuille de près d'un mètre de hauteur), l'ensemble de la page, dans ce médium léger qui autorise encore les repentirs : le crayon graphite.

$\mathrm{Ne}$ reste plus ensuite qu'à encrer la page. Cette opération de mise au net lui confère son aspect définitif et propre à la reproduction. "Le stade d'achèvement du crayonné connaît toutes sortes de graduations, entre la simple esquisse sommaire et le dessin poussé, où ne manquent pas une ombre, pas un détail, pas une hachure. Dans le premier cas, le crayonné ne va pas au-delà d'une mise en place et c'est véritablement au stade de la mise à l'encre que le dessin naît sur le papier, qu'il s'invente, prenant figure et consistance. Dans le second, l'encrage est exécution minutieuse, appliquée, dépourvue d'autonomie ${ }^{10}$.»

L'encrage se faisant par-dessus le crayonné, celui-ci disparaît en tant que tel, car il est entièrement recouvert. Les quelques traces de crayon qui peuvent encore apparaître seront gommées. Ainsi le crayonné est-il un document d'étape qui, en règle générale - et contrairement aux esquisses qui, elles, ont été réalisées sur des feuilles séparées -, ne survit pas à l'achèvement de la planche, mais s'efface sous l'encrage palimpseste.

Les crayonnés sont donc des objets rares. Ils ne survivent sous cette forme que lorsque le dessinateur a abandonné la planche, ou s'il a pour méthode d'encrer sur une autre feuille, soit par transparence, soit en y reportant son crayonné au moyen d'un calque. Cette façon de procéder était celle d'Hergé, qui l'avait adoptée à l'instigation de son ami et confrère Edgar P. Jacobs.

La rareté des crayonnés fait tout leur prix, d'autant qu'aux yeux du connaisseur, c'est véritablement à ce stade que peut encore pleinement s'apprécier un dessin encore libre, jaillissant, inventif et sensuel. Le critique Pierre Sterckx est lyrique : «Les crayonnés d'Hergé sont très exactement dans le même rapport avec ses mises à l'encre (et les imprimés qui s'en suivent) que les lavis de Poussin ou les dessins de Monsieur Ingres avec leurs tableaux ${ }^{11}$.»

9. Voir Art Spiegelman, MetaMaus, Paris, Flammarion, 2012.

10. Je cite ici mon article «Encrage», «Dictionnaire esthétique et thématique de la bande dessinée», op. cit., avril 2014 : <http://neuviemeart.citebd.org/spip.php?article766>.

11. Notice dans le catalogue de la vente Artcurial du 8 juin 2013, «L'univers du créateur de Tintin», p. 38. Ces lignes présentaient le crayonné de la planche 32 de Tintin au Tibet. Estimé entre 140000 et 160000 euros, il fut adjugé pour 189500 euros. 
La planche originale, dans son état final (encré), montre-t-elle autre chose que la page correspondante telle qu'elle paraitra dans un magazine ou un livre ? C'est selon. Comme l'a écrit Jean-Pierre Mercier, «certains, comme Gotlib, André Franquin ou Joost Swarte, fournissent des pages qui sont, à quelques rares et minimes retouches près, exactement conformes à ce qu'elles seront une fois imprimées. [...] D'autres, au contraire (Alberto Breccia, Jean-Claude Forest...) conçoivent la page comme un lieu d'expérimentation et conçoivent de multiples grattages, collages, projections, sans garantie d'un résultat imprimé complètement satisfaisant, car le processus de reproduction peut trahir certaines de ces tentatives virtuoses ${ }^{12}$.»

Il n'est pas rare que l'original soit agrémenté de croquis dans les marges. On trouve aussi parfois (notamment chez les dessinateurs du journal Spirou des années soixante et soixante-dix), au verso, des couleurs grossièrement posées, qui serviront d'indications au studio de coloriage.

L'Américain Craig Thompson (Blankets, Habibi) va jusqu'à tenir une sorte de journal au verso de ses planches. Il y note ce qu'il a fait le jour de leur exécution, la météo du jour, s'il a été interrompu dans son travail, etc.

Du point de vue de la génétique, le plus intéressant, ce sont les traces encore visibles sur la planche qui témoignent de son exécution même. Les dessinateurs utilisaient volontiers autrefois un crayon bleu plutôt qu' un noir, car cette couleur ne «passait» pas lors de la photogravure. Il n'était donc pas nécessaire de gommer. Des rehauts de bleu pouvaient aussi indiquer l'emplacement des zones qui seraient grisées ensuite au moyen de trames d'imprimerie. Les repentirs, eux, prennent la forme de retouches à la gouache blanche (elles foisonnent sur les originaux d'Alain Saint-Ogan, le père de Zig et Puce) ou au correcteur liquide, mais aussi de collages ou de «rustines» (case découpée, remplacée par une autre qui a pris sa place). Il est arrivé à Moebius de refaire une case et de la fixer au moyen d'une charnière mobile, garantissant ainsi un accès à la version initiale qui se trouve dessous, et la possibilité de comparer les deux.

Pour imprimer ses albums (sept titres publiés dans les années 1830 et 1840), Töpffer utilisait un papier dit «autographique», c'est-à-dire un papier de report, qui lui permettait de transposer à l'envers sur la pierre lithographique des dessins et des mots tracés dans le bon sens, à la plume ${ }^{13}$. Les planches ainsi réalisées ne survivaient pas au processus de fabrication. Seules les pierres (qui n'ont pas été conservées) permettaient de réaliser un nouveau tirage.

En revanche, il existe pour chacun des albums un brouillon complet, dessiné dans un carnet oblong, et ces versions préliminaires, elles, sont conservées dans les collections publiques genevoises. Pour passer du brouillon à la version imprimée, Töpffer se recopiait en apportant un certain nombre de changements et d'améliorations, ajoutant ou soustrayant quelquefois des scènes entières. La comparaison entre les brouillons et les versions autographiées est passionnante et riche d'enseignements sur la science töpfférienne du découpage ${ }^{14}$. Ainsi que l'a noté Walter Lindner, les suppressions, ajouts et autres retouches pratiqués « révèlent

12. «Un objet incertain : l'original de bande dessinée», op. cit., p. 26.

13. Il fut l'un des très rares artistes à utiliser ce procédé. Pour plus de détails, je renvoie à mon essai $M$. Töpffer invente la bande dessinée, Bruxelles, Les Impressions nouvelles, 2014, p. 73-81.

14. Le cas de l'Histoire de Mr Cryptogame est particulier, puisque la santé déclinante de Töpffer ne lui permit pas de réaliser la version destinée à l'impression. Cham la grava d'après ses brouillons, pour L'Illustration . 
un véritable travail de metteur en scène dans lequel on distingue des efforts qui concourent à l'accélération ou au ralentissement de l'action ». Et il n'est pas de meilleur objet, pour cette étude, que l'album intitulé Histoire d'Albert (1845), dont le «brouillon » comporte déjà, à lui seul, jusqu'à trois couches de corrections de dessins collées les unes sur les autres, cas unique dans tout le corpus töpfférien 15 .

On l'a dit, certaines bandes dessinées ont connu des ajouts, repentirs et autres modifications après qu'elles ont été diffusées dans le public, au gré des changements de support ou des rééditions. L'Américain Chris Ware, éternel insatisfait, a ainsi ajouté deux pages à la fin de son très remarqué Jimmy Corrigan ${ }^{16}$, quelques mois seulement après sa sortie.

Les variantes entre les versions successives de certaines des Aventures de Tintin ont été particulièrement étudiées. Le destin éditorial du diptyque composé des 7 Boules de cristal et du Temple du soleil avait été quelque peu chahuté par la Seconde Guerre mondiale. Commencé dans le quotidien Le Soir sous la forme de bandes en noir et blanc (de septembre 1943 à septembre 1944), le récit avait repris après deux années d'interruption dans le tout nouvel hebdomadaire Tintin, dont il occupait alors la double page centrale, utilisée comme un espace horizontal homogène. Hergé dut «réaliser parallèlement deux "montages" de l'histoire : l'un destiné à l'édition en album et limité à soixante-deux pages verticales; l'autre conçu pour le journal et proposant un nombre libre de planches en format "à l'italienne" ». Comme on peut l'observer en comparant les éditions courantes avec l' «édition originale» du Temple du soleil publiée en 1988 dans la «Bibliothèque de Moulinsart», « le passage à quatre bandes au lieu de trois et le retour au format vertical [furent] l'occasion de multiples transformations, allant du changement de lettrage à la suppression de vignettes redondantes en passant par les recadrages, les remises en couleurs et les améliorations de dialogue ${ }^{17}$.

L'Île noire a, de son côté, connu une version en noir et blanc en 1938, puis une première version en couleurs en 1943, et une seconde, entièrement redessinée, en 1966. Ludwig Schuurman a consacré sa thèse, soutenue en 2009, à l'étude comparative des trois versions, concluant à une édulcoration et un affadissement du récit dans la troisième, celle que nous connaissons.

Une approche de la bande dessinée en termes de critique génétique peut aussi être étendue, pour une large partie de la production, au réseau intertextuel qui se tisse à l'intérieur d'une série donnée («les aventures de...»). D’un épisode à l'autre, on voit un univers se construire, s'enrichir, se stabiliser, une esthétique s'affirmer, un «cahier des charges » propre à la série se mettre en place. Ce processus diachronique d'invention d'un univers fictionnel dans la durée n'est sans doute pas le moins passionnant des champs ouverts à l'étude.

15. Voir Walter Lindner, «L'Histoire d'Albert de 1845 : réflexions sémiotiques et idéologiques », dans Propos töpffériens, Chêne-Bourg/Genève, Georg/Société d'études töpffériennes, 1998, p. 97-108 (si le titre de l'article est en français, le texte, lui est en allemand). Pour des précisions sur les changements opérés par Töpffer sur l'ensemble de ses histoires, voir les «notes et références » de David Kunzle, à la fin de son édition intégrale en langue anglaise : Rodolphe Töpffer, The Complete Comic Strips, Jackson, University Press of Mississippi, 2007, p. 627-650.

16. Jimmy Corrigan, The Smartest Kid on Earth, New York, Pantheon Books, 2000.

17. Les deux citations proviennent de l'introduction, non signée, à cette édition originale de 1988. 
THIERRY GROENSTEEN est directeur de collection pour Actes Sud et chargé de mission à la Cité internationale de la bande dessinée et de l'image. Rédacteur en chef de la revue en ligne NeuvièmeArt 2.0, il est l'auteur de nombreux ouvrages sur la bande dessinée, parmi lesquels Système de la bande dessinée (PUF, 1999), Un objet culturel non identifié (L'An 2, 2006), La Bande dessinée, son histoire et ses maîtres (Skira/Flammarion, 2009), Bande dessinée et narration (PUF, 2011) et Un art en expansion (Les Impressions nouvelles, 2015).

thierry.groensteen@wanadoo.fr

\section{La fabrique de la bande dessinée}

Au confluent de la littérature et des arts visuels, la bande dessinée est tantôt l'œuvre d'un auteur unique, tantôt le résultat d'une collaboration entre un spécialiste du texte et un spécialiste de l'image. De l'écriture du scénario à sa mise en dessin, le processus de réalisation d'une œuvre suit un protocole qui connaît de nombreuses variantes et peut s'incarner dans des documents d'étape de formes multiples. Il est difficile d'en trouver un qui corresponde à la définition du manuscrit autographe de l'écrivain. La planche originale est l'objet le plus emblématique de ce processus, et celui qui a acquis, depuis une vingtaine d'années, une valeur marchande sur le marché de l'art. Mais une génétique de la bande dessinée doit s'intéresser à tout ce qui se déroule en amont de l'exécution de la planche, aussi bien qu'à ce qui peut se produire en aval : ajouts, repentirs, modifications au gré des changements de support ou des rééditions.

A meeting place of literature and the visual arts, the comic strip is either the work of a single author or the result of a collaboration between a text specialist and an image specialist. From the writing of the scenario to its illustration, the process of making a work follows a protocol that has many variants and can be materialized in several kinds of documents for each stage. It is difficult to find one that matches the definition of the writer's autograph manuscript. The original plate is the most emblematic object of this process and the one that in the past twenty years has acquired a sales value on the art market. But a genetic study of comic strips has to examine all that happens before the plate is drawn, as well as what can happen afterward: additions, corrections, modifications that vary according to medium changes or re-editions.

Im Verschmelzen von Literatur und visuellen Künsten ist der Comic mal das Werk eines einzelnen Autors, mal das Resultat einer Zusammenarbeit zwischen einem Text-Spezialisten und einem Bild-Spezialisten. Vom Schreiben des Szenarios bis zur Umsetzung in einer Zeichnung verläuft der Prozess der Realisierung eines Werks nach einem Protokoll, das zahlreiche Varianten kennt und in den vorläufigen Dokumenten vielfältige Formen annehmen kann. Es ist schwierig, darunter ein Dokument zu finden, welches dem handschriftlichen Manuskript eines Schriftstellers entspricht. Die ursprüngliche Bildtafel ist das symbolträchtigste Objekt in diesem Prozess, und auch jenes, welches seit etwa zwanzig Jahren, einen kommerziellen Wert auf dem Kunstmarkt erworben hat. Aber eine Genese des Comics muss sich für all das interessieren, was sich vor der Ausführung der Bildtafel abspielt, ebenso für all jenes, was danach abläuft: Hinzufügungen, Abänderungen, Umgestaltungen je nach Trägersubstanz oder bei Neuauflagen.
En la confluencia de la literatura y de las artes visuales, la historieta puede ser tanto la obra de un solo autor, como el resultado de una colaboración entre un especialista del texto y un especialista de la imagen. De la escritura del guión hasta su realización gráfica, el proceso de construcción de una obra sigue un protocolo que comporta numerosas variantes y puede encarnarse en documentos de etapa de diversas formas. Resulta difícil encontrar entre ellos uno que corresponda a la definición del manuscrito autógrafo de escritor. La lámina original es el objeto más emblemático de este proceso y ha adquirido, en los últimos veinte años, un valor mercantil en el mercado del arte. Pero una genética de la historieta tiene que interesarse tanto en lo que precede la realización de la lámina como en lo que puede producirse después: añadidos, arrepentimientos, modificaciones ligadas a los cambios de soporte $\mathrm{o}$ a las reediciones.

$\mathbf{N}$ a confluência da literatura e das artes visuais, a banda desenhada tanto pode ser obra de um autor único, como resultado de colaboração entre especialistas do texto e da imagem. Da redação do roteiro até à sua passagem ao desenho, a produção da obra segue um protocolo com inúmeras variações e diversas etapas. Mas é difícil encontrar uma correspondência para a definição do manuscrito autógrafo do escritor. A chapa original é o objeto mais emblemático do processo, e adquiriu, desde há vinte anos, valor comercial no mercado de arte. Mas a genética da banda desenhada deve interessar-se também por tudo o que está antes, bem como por tudo o que vem depois: adições, alterações, mudanças no suporte ou reedições.

A 1 crocevia tra letteratura e arti visive, il fumetto è talvolta l'opera di un unico autore, talvolta il risultato di una collaborazione tra uno specialista del testo e uno specialista dell'immagine. Dalla scrittura del testo al suo disegno, il processo di realizzazione di un'opera segue un protocollo che subisce numerose varianti e può incarnarsi in documenti transitori di forme differenti. È difficile trovarne uno che corrisponda alla definizione di "manoscritto autografo" dello scrittore. La tavola originale è l'oggetto più emblematico di questo processo ed è quello che ha acquistato, da una ventina d'anni, un valore commerciale sul mercato dell'arte. Ma una genetica del fumetto deve interessarsi a tutto ciò che si sviluppa sia a monte dell'esecuzione della tavola, sia a valle: aggiunte, ripensamenti, modifiche, in seguito ai cambiamenti di supporto o alle riedizioni. 